\title{
Experiential and Integrated Learning Environments - Teaching Urban Design Studio at Curtin University
}

\author{
Francesco Mancini, Tanja Glusac
}

Department of Architecture, Curtin University, Western Australia.

\begin{abstract}
Experiential and Integrated Learning Environments in Architecture is an educational project based on action learning pedagogy (Revans, 1980) that challenges the traditional design studio teaching approach to Architectural/Urban Design and builds on Dewey (1939) and Kolb (1984) theories of experiential learning. An innovative model of teaching Urban Design to Master of Architecture students has been trailed for the first time in 2018, when the studio was set in the City of Bayswater, and has been refined over the course of 2019 in two separate study periods - Study Period 1 (Rome/Milan Study Tour) and Semester 2. This model provided students with an opportunity to collaboratively learn from and re-design the existing urban environments by immersing themselves in the very context they are studying. The proximity of the classroom to the urban setting presented an opportunity for students to draw comparisons and analysis between national and international examples and that of the surrounding urban milieu. Additionally, advanced technology supportive of distributed learning environment and intense collaboration with industry such as Hassell, Element and The Office of the Government Architect (OGA), coupled with opportunities to visit various practices, provided deeper insights and an all rounded approach to learning and engaging with architecture.
\end{abstract}

Keywords: experiential learning; collaborative learning environments; architecture; urban design. 


\section{Learning in traditional environment vs experiential learning in Architecture}

Research proves that ability to work and design in teams is a fundamental skill for creative professions (Hirsh and McKenna, 2008). Architecture in particular requires collaboration across various disciplines and an ability to synthesize the built form from a large number of inputs. The design-by doing approach, which architecture largely utilizes in a specific learning environment traditionally defined as 'the studio', resonates with a pedagogy based on experiential learning.

We consider learning environment as a combination of factors contributing to student learning. In line with The Glossary of Education Reform (2013), "Learning environment refers to the diverse physical locations, contexts, and cultures in which students learn... The term also encompasses the culture of a school or class - its presiding ethos and characteristics, including how individuals interact with and treat one another".

Experience is central to both learning and the environment in which learning takes place. Dewey (1939) and Kolb's (1984) concept of experience, described as "the process whereby knowledge is created through the transformation of experience" (Kolb, 1984, 38) complements the learner-centred approach desired in contemporary education. Kolb's fourstage learning cycle consisting of concrete experience, reflective observation, abstract conceptualization and active experimentation focuses on periodically exposing learners to all four phases, linking each phase with one another rather than prioritising any of them (Healey and Jenkins 2000). This enables students to consider each stage of their learning as part of a continuum, which corresponds to their growth in theoretical knowledge, practical skills, creative applications and a life-long collaborative approach. Kolb's vision of experience presents analogies to the Design process as it aligns with constructivism (Scheer, 2012), which enables teaching design through the experiential learning theory taking advantage of a composite and diverse learning environment.

In Urban Design Research Studio units offered at Curtin University we challenged the traditional learning environment of Architectural Design units, which according to Lawson (2019) constitutes of studio space, design library, design tutorial run by academic staff and the design critiques conducted by industry guests and academics. In particular, we challenged Lawson's idea that traditional 24/7 studio culture is de facto irreplaceable due to the variety of teaching activities and informal interactions amongst staff and students which stimulate creative thought in such an environment. The growing reduction of resources in the higher education ${ }^{1}$ increasingly challenged this studio structure that was used by most Architecture

\footnotetext{
${ }^{1}$ Berg and Seeber (2016) offer a comprehensive analysis of current restrictions brought by reduction of time, space and human resources in delivering higher education. Reflections on the impact and consequences these reductions have on architectural education are outlined in Mancini and Glusac (2018) 'From time to Time: A constructivist approach to sociality in learning'.
} 
Schools for decades to simulate a typical XX century office practice. We responded to this problem by replacing the time-consuming master-apprentice model through the proposition of a project based on team work and experiential and integrated learning environment.

The two units were offered in two different contexts and delivery modes - a six weeks intensive travel Design Studio unit of Rome and north-central Italy in Study Period 12019 and a regular semester based studio run from the former Perth Technical College, $137 \mathrm{St}$ Georges Terrace, Perth Central Business District (CBD) in Semester 2 2019. The Semester 2 iteration maintained the core principles of design learning processes consisting of learning by doing rather than through formal instructions. In this sense, the main innovation in comparison with traditional studio is the replacement of individual studio desk critique with emerging collaborative activities extracted from contemporary practice. This pedagogic strategy allowed students to question the assigned design problem through iterative propositions of possible solutions (Lawson 2006; 2019) while being stimulated by opportunities to immerse themselves in the context they are studying - a hybrid setting comprising of blended real on-site experience and digital learning environment.

Students, organized in teams of five or six, engaged in inquiry by design research to support an urban Design project through current theories of urbanism and explored integrated sustainable approaches to dense dwelling and urban mixed-use, especially at the 'groundfloor' of the city. In line with the Government of Western Australia's commitment to improve the quality of built environment, we co-designed the units with practitioners from Hassell, Element and OGA adopting "the objectives, measures, principles and processes which apply to the design and assessment of built environment proposals through the planning system" as set by the Design WA State Policy in 2019 (Department of Planning, Lands and Heritage, 2019, 3). Work Integrated Learning approach in these units follows the practice-based multidisciplinary Design Review. The robust industry partners' support and engagement culminated in a successful final presentation of student work at Hassell.

\section{Experiential and Integrated Learning Environments in Architecture}

Bearing in mind that Masters students are expected to take responsibility and autonomy for their learning, the teaching team progressively designed Experiential and Integrated Learning Environments in Architecture project on four fundamental principles:

1. engagement with the perceptual experience of the physical built space of the city and its abstract conceptualisation, also the subject of learning, as alternative to inclass lectures and the study of the built environment through literature;

2. work in classroom expressed through the substitution of traditional one-on-one desk critiques in studio with a collaborative methodology of work supported by robust work ethic procedures producing and exchange knowledge to complement tutorials; 
3. Expanded learning environment by extending the learning design and experience through flipped classroom, engagement and collaboration with industry and international academics, including fish-bowl techniques;

4. incremental integration of traditional and advanced digital technology methods enabling guided access to online information, examination of unit digital resources and real time design constructive critique.

We based the entire learning project on real industry practices and processes to increase its coordination, implementation and evaluation, which offered us a sound model to align the scope of the unit with studio delivery practices, assessment briefs and marking rubrics. More specifically, in both Study Period 1 (SP1) and Semester 2 2019, students worked in small teams on urban design proposals and individually on building design. The teams worked on developing intense didactic learning activities aimed at developing a historical, morphological and experiential analysis which inform a complete and coherent urban and architectural proposal integrating design at various scales. To that end, they employed a variety of qualitative research methods such as site visits and data collection, sketching, drawing, photography, and writing to reflect on experiences of the studied urban context, to produce and communicate advanced $2 \mathrm{D}$ and $3 \mathrm{D}$ design concepts. The integration of the digital (e.g. Blackboard) and physical (e.g. Studio space) environments as parts of the same learning space, in which formal, informal contact time and non-contact time are part of a continuous learning experience was deemed essential as it expands the learning environment beyond the classroom.

Further to this, in Semester 2 we introduced a model of assessment as learning, whereby two weeks prior the assessment submission students presented to a multidisciplinary industry panel who assessed the presentation of each team's project against Design WA criteria and discussed the formative feedback with the panel members, as confirmed by Tanya Ring, OGA, Guest lecturer and critic: "The Office of the Government Architect (OGA) was supportive of the decision to include the Design WA planning policy suite within the curriculum for this studio... It provided students with experience in presenting in front of a panel tasked with assessing projects against the same Design Principles that current Design Review Panels ... use for ascertaining design quality in the planning system."

The practice of integrating Design WA framework further enabled the establishment of thresholds of students' work against national and international academic and practice-based benchmarks by defining learning outcomes, assessment briefs and marking descriptors in line with the National Standards of Competencies for Architects, making the link between education and practice standards explicit and benchmarked by the industry itself. 


\section{Project Implementation}

Architectural Design is about the production of built form in context, which is not just physical, urban or rural; it involves the comprehension of social, historical and cultural background of its inhabitants as well as future users, which the two studios aimed to achieve. Within this pedagogic vision, students enrolled in Rome and Perth Urban Design Studios benefited from the integration of different learning environments in a number of ways. The indispensable physical experience of the place was supplemented by digital technology enabling students easy access to historical maps and information. Together they allowed for a comprehensive understanding of the site's development to be traced.

In SP1, the Rome Studio offered "A great opportunity to understand how an urban architectural intervention can acknowledge the various physical and intangible conditions of the city at different scales... [and] how the history and the urban fabric transformations of the place can influence or underpin design." ... "The constant feedback and site visits throughout Italy ... informed how we thought, experienced and expanded our knowledge...[which in] turn informed our design and approach to the assignments and design for site." (eVALUate ARCH5024 2019 SP1)
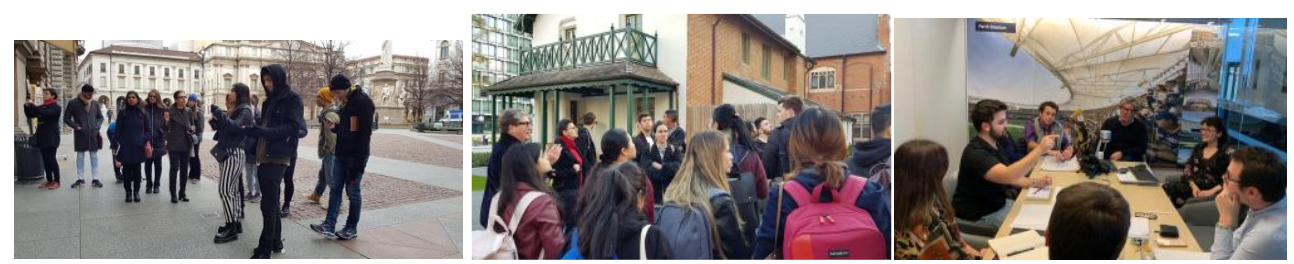

Figure 1-2. The city as an open book: Milan and Perth.. Figure 3. Critique with Hassell Architecture at their office.

Building on the experience of the travel studio, in Semester 2 2019, Urban Design Research Studio was taught from the Technical College in Perth CBD utilizing once again the direct experience of the built environment as an open-air book. To this end, six site visits in the city of Perth substituted the in-class lectures and integrated the assigned readings. The studio placed an emphasis on visual analysis expecting of students to provide evidence of their reading via drawings and diagrams of the visited places substituting the traditional literature review.

The very presence in the city centre augmented the learning experience of this urban design studio, which was further supplemented through advanced technology available on the premises. The venue at St Georges Terrace within which we worked, consists of 6 screens that were used to project information from 4 different sources at the same time. The teaching team also connected an iPad with Apple pencil to the room screen system as one of the input sources. This made it possible to capture images from different screen projections showing the project site, precedents and students' diagrams which tutors could comment through 
digital drawings in real time and send these to students/team via email or social media platforms for their further reflection. Students' sketches were scanned and projected on screen to comment on ideas and strategies. The available technology offered high potential for augmenting tutorials as these could be recorded and distributed synchronously and asynchronously. This process replaced the master-apprentice model based on one-on-one feedback and enabled everyone to share the traditional 'drawing conversation', typical of the one-on-one desk critique, with the whole class. Such an advanced simulation of a project critique, typical of professional practice, extends both participation and feedback's impact to the whole class/cohort, evident in the following statement: "The Curtin campus in Brookfield Place made the unit all the more interesting and motivational. The technological facilities and amenities provided to us ensured that we were always well equipped and able to interact, learn and become more of a part of the teaching / learning process." (ARCH5024-25 students' LinkedIn testimonials).

\section{Impact of engagement - linking education, practice and digital technology}

Diversifying the whole-day studio sessions increased students' participation and enabled students with different learning styles (Kolb 1984) to equally participate and engage in learning while building their own learning platforms to process design ideas in a manner original to them. The results of redesigning the learning experience through a new learning environment have been very positive as demonstrated by students' comments: "The most helpful aspects have been the overall learning environment. Studying in the city has been fantastic and has allowed for lots of learning opportunities, networking and site visits. The meetings with other people in the profession meant that we had mini deadlines, and this helped keep us on track and allowed us to create as much work as we did. [Tutors] put in a lot of effort to make the semester great... [It] has been by far my favourite semester for studio and methods!" (ARCH5024 eVALUate Semester 2 2019) and industry's confirmation of the high quality of students' learning: "The challenge of teamwork was embraced by the students, maintaining respectful relationships and balancing divergent 'personalities' to achieve a valuable life lesson and a positive learning outcome... The project brief was huge in scale and complexity and the students were fearless in tackling specific areas that allowed individual development of their ideas, whilst still being grounded in the group's common masterplan vision, no easy feat." (Architect Jane Wetherall, industry guest). 
Table 1. Maps activities and indicators against the four fundamental principles used to design Experiential and Integrated Learning Environments in Architecture project.

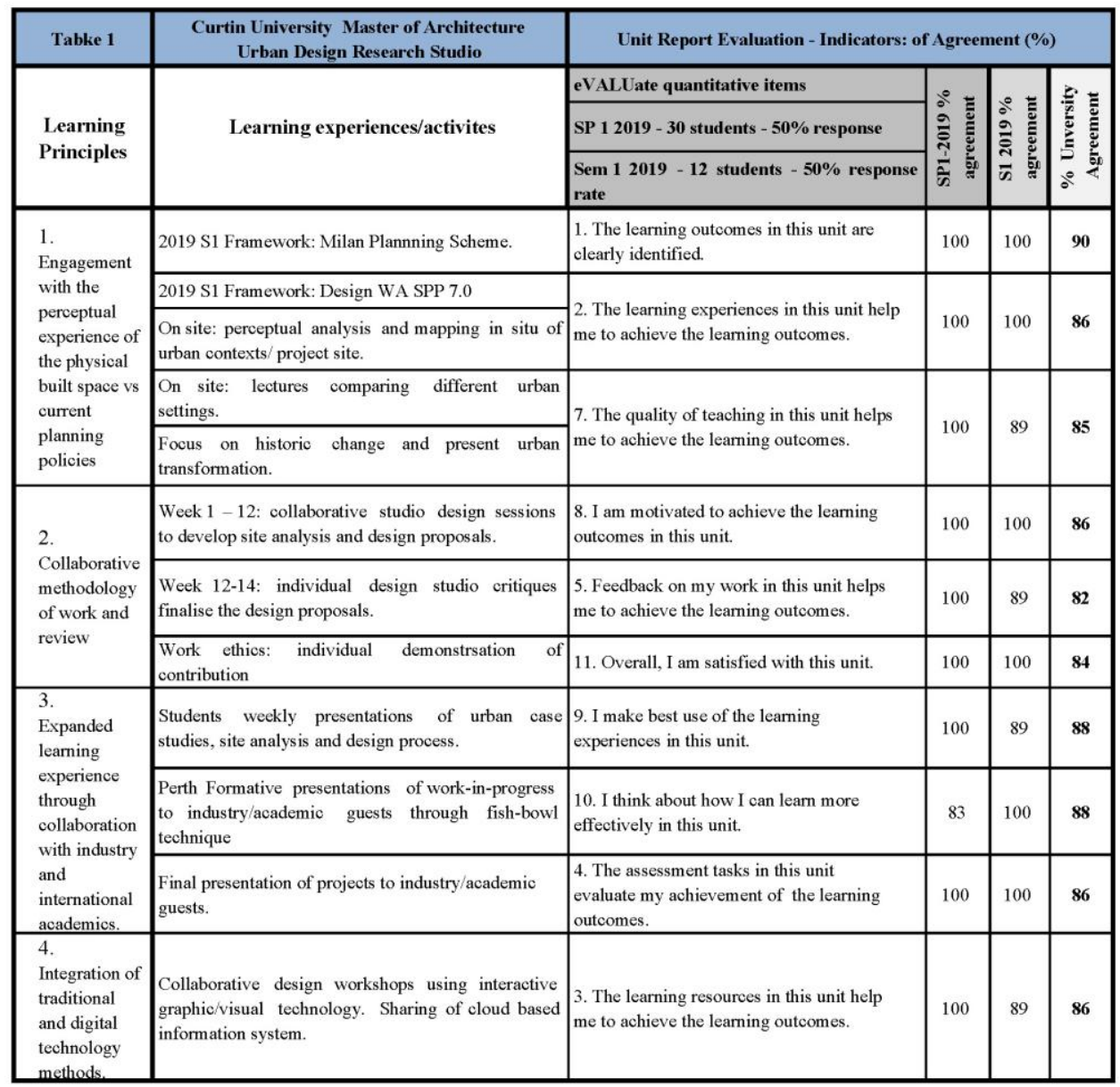
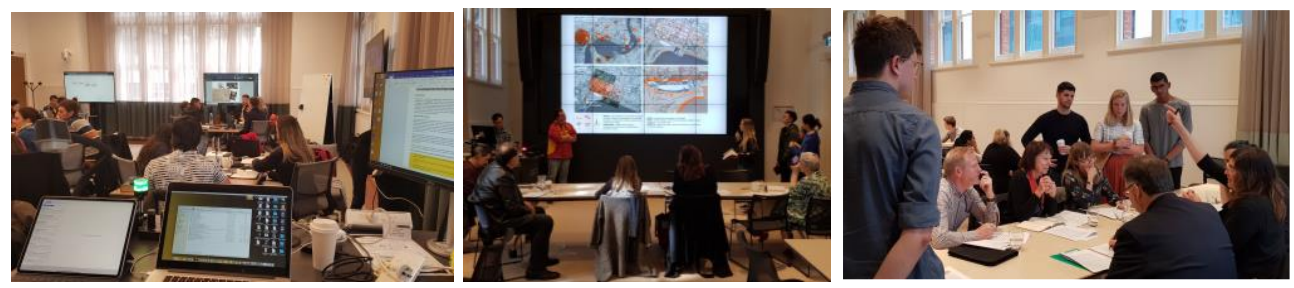

Figure 4. digital studio setting Figure 5. Industry Design Review Panel

Figure 6. Fish-bowl Technique 
Similarly, the Rome Study Tour took advantage of extensive site visits to a number of different cities in north-central Italy and lectures delivered on site to demonstrate, via built examples, concepts and theories of the assigned readings, as evident in the following statement: "Physically experiencing architecture should be an essential part of this course, as I have learnt so much more than a lecture on a particular city in Italy. My motivation was unwavering, through the critical knowledge of both [tutors], I was genuinely excited each day to uncover new layers of meaning within the city of Rome. Additionally going through the studio as a group was very important as the critical analysis was perpetuated through discussion." (eVALUate ARCH5024 2019 Study Period 1)

\section{Conclusion}

Educating future designers to make responsible choices is a point of difference to endow a sustainable built environment for future generations. Research proves that using different formal manipulation techniques develops designers' (and students) curiosity, empathy and lateral thinking when looking for Design solutions (Brown, 2008; Munari, 1971 and 1977). The Experiential and Integrated Learning Environments in Architecture project afforded us the opportunity to explore innovative ways of teaching Urban Design to Architecture students utilizing both physical presence and digital technology to augment student experience.

In the end, reflecting on students' qualitative responses, the project, though a success, proved that traditional studio critique can largely be replaced by up 70\% of the classes through innovative collaborative learning experiences. Nevertheless, individual critiques are still an essential component in the later part of the architectural design process. With this in mind we intend to refine the project further and offer it again, for it has impacted greatly on student learning and experience and effectively contributed to the increase in overall students satisfaction which moved from 75\% in 2018 (Bayswater studio) to 100\% received in both Study Period 1 and Semester 2019.

\section{References}

Berg, M and Seeber, B. (2016). The Slow Professor: Challenging the Culture of Speed in the Academy. Toronto: University of Toronto Press.

Brown, Tim, (2008) Design Thinking. Harward Business Review, June,86-92

Curtin eVALUate (2019). Urban Design Research Studio Study Period 1 and Semester 2.

Department of Planning, Lands and Heritage. 2019. State Planning Policy 7.0 Design of The Built Environment. Perth: Western Australian Planning Commission.

Dewey, J. (1938). Experience and education. New York: Macmillan. 
Hirsh, P. and McKenna F. (2008). Using Reflection to Promote Teamwork Understanding in Engineering Design. Education International Journal of Engineering Education, 24(2), 377-385.

Kolb, D. A. (1984). Experiential learning: experience as the source of learning and development. Englewood Cliffs, NJ: Prentice Hall.

Mancini, F and Glusac T (2018) From time to Time: A constructivist approach to sociality in learning, 4th International Conference on Higher EducationAdvances (HEAd'18) conf. proceedings, 2018, pp.1567-1577

Munari B. (1977). Fantasia. Laterza: Bari.

Munari, B. (1971) Design ans Art, penguin Book: london

Revans, R. (1980). Action learning: New techniques for management. London: Blond \& Briggs, Ltd.

The Glossary of Education Reform. (2013). https://www.edglossary.org/learningenvironment/ (02.12.2019).

ARCH5024-25 students' LinkedIn testimonials.

All images taken by authors. 